\title{
(18)F-sodium fluoride PET in multiple myeloma: Linking cancer to atherosclerosis?
}

\author{
Dominik C. Benz, MD, ${ }^{\mathrm{a}, \mathrm{b}}$ Ronny R. Buechel, $M D,{ }^{\mathrm{a}}$ and Sharmila Dorbala, MD, \\ $\mathrm{MPH}, \mathrm{FASNC} \mathrm{C}^{\mathrm{c}, \mathrm{d}, \mathrm{e}}$ \\ a Department of Nuclear Medicine, Cardiac Imaging, University Hospital Zurich, Zurich, \\ Switzerland \\ b Department of Cardiology, University Heart Centre, University Hospital Zurich, Zurich, \\ Switzerland \\ c Amyloidosis Program, Division of Cardiology, Department of Medicine, Department of \\ Radiology, Brigham and Women's Hospital, Boston, MA \\ d Cardiovascular Division, Department of Radiology, CV Imaging Program, Brigham and \\ Women's Hospital, Boston, MA \\ e Division of Nuclear Medicine, Department of Radiology, Brigham and Women's Hospital, \\ Boston, MA
}

Received Sep 9, 2020; accepted Sep 9, 2020

doi: $10.1007 / \mathrm{s} 12350-020-02447-z$

\section{See related article, pp. 3044-3054}

Multiple myeloma (MM) is a hematologic malignancy of plasma cells and is characterized by end-organ damage (e.g., hypercalcemia, renal dysfunction, anemia, or lytic bone lesions) due to the proliferation of a single plasma cell clone. It is assumed to evolve from an asymptomatic premalignant stage without end-organ damage and less than $10 \%$ of monoclonal plasma cells in the bone marrow (i.e., monoclonal gammopathy of unknown significance, MGUS). If monoclonal plasma cells exceed $10 \%$, but end-organ damage is absent, smoldering multiple myeloma (SMM) is diagnosed. All these gammopathies (MM, MGUS, and SMM) might progress to systemic immunoglobulin light-chain amyloidosis (AL) when the circulating monoclonal light chains are deposited as amyloid fibrils in the extracellular tissue-most commonly affecting the kidneys and the heart. Approximately $10 \%$ to $15 \%$ of patients with MM will have evidence of cardiac amyloidosis. ${ }^{1}$

Reprint requests: Dominik C. Benz, MD, Department of Nuclear Medicine, Cardiac Imaging, University Hospital Zurich, Ramistrasse 100, 8091 Zurich, Switzerland; dominik.benz@usz.ch

J Nucl Cardiol 2021;28:3055-7.

$1071-3581 / \$ 34.00$

Copyright (C) 2021 American Society of Nuclear Cardiology.
The risk of adverse cardiac events, mainly arrhythmia and congestive heart failure, is doubled in patients with MM. ${ }^{2}$ Primarily, this excess in cardiac risk is accounted for by the disease-related risk from cardiac $\mathrm{AL}$ amyloidosis and the risk from treatments associated with cardiovascular toxicity (e.g., doxorubicin, proteasome inhibitors, and immunomodulators) including thrombotic complications. ${ }^{3}$ On the contrary, the annual rate of ischemic events is about $10 \%$-with no differences between patients with and without MM. Therefore, the increased ischemic risk has not been linked to MM per se, but rather to the high prevalence of age-related co-morbidities in this elderly patient population affected by MM (Figure 1).

The acceleration of atherosclerosis in patients with MM nevertheless, might have been underestimated in light of the poor prognosis of the disease and is supported by two clinical features of MM. First and foremost, up to $22 \%$ of patients with MM have been reported to carry an expanded somatic blood-cell clone, ${ }^{4}$ an entity labeled clonal hematopoiesis of indeterminate potential (CHIP), which has previously been associated with a doubling in the risk of coronary artery disease. ${ }^{5}$ Second, as outlined above, MM features hypercalcemia that could drive vascular microcalcification if vascular uptake was increased. Fortunately, the metabolic process of microcalcification can be visualized by ${ }^{18} \mathrm{~F}$-sodium fluoride $(\mathrm{NaF})$ positron emission tomography (PET), and there is emerging evidence that NaF PET confers prognostic value in that it predicts ischemic events, with 


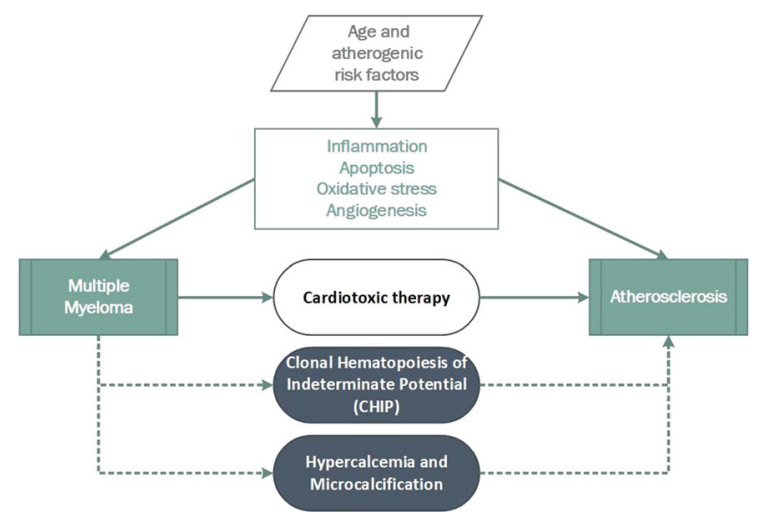

Figure 1. Potential mechanisms linking multiple myeloma and atherosclerosis.

an over sevenfold increase of fatal and nonfatal myocardial infarctions in patients with increased coronary $\mathrm{NaF}$ uptake. ${ }^{6} \mathrm{NaF}$ PET also proved valuable in extracoronary atherosclerosis: in patients with abdominal aortic aneurysms, aortic NaF uptake identified aneurysms that expanded 2.5-times more rapidly and were nearly three-times more likely to rupture or undergo surgical repair. ${ }^{7}$ Given the high prevalence of CHIP and hypercalcemia, translating NaF PET to patients with MM could enhance our pathophysiological understanding of the mechanisms linking cancer to accelerated atherosclerosis.

In the current issue of the Journal, Arani and colleagues compared $\mathrm{NaF}$ uptake in 44 patients with $\mathrm{MM}$ and SMM from the FULIMA cohort ${ }^{8}$ to 26 age- and gender-matched healthy controls from the CAMONA cohort. ${ }^{9}$ The mean of the standardized uptake value (SUVmean) of $\mathrm{NaF}$ was measured in the ascending aorta, the aortic arch, and the descending aorta, and in successive axial slices of the heart to calculate wholeheart uptake. Target-to-background ratios (TBR) were increased significantly in all areas. Interestingly, however, it only correlated with the extent of calcifications (as measured by calcium score, calcium volume, and calcium mass) in the aortic arch, but not in the ascending or descending aorta. The authors concluded that $\mathrm{NaF}$ uptake is higher in patients with MM, and NaF-PET could be an appealing method to better characterize the very early disease process of atherosclerosis in these patients.

The authors should be congratulated for "thinking outside the box" by highlighting and elaborating on the fascinating interrelation between hemato-oncology and atherosclerosis. The study suggests that atherosclerosis may be more "active" in patients with MM, and stringent risk factor control could be critical. Furthermore, the poor correlation of $\mathrm{NaF}$ uptake with the morphological manifestation of atherosclerosis (i.e., calcifications) strengthens the demand for novel functional tools to stratify patients at risk for coronary artery disease. Ultimately, in the larger context, the findings indicate that cancer may indeed accelerate atherosclerosis, questioning - at least in patients with $\mathrm{MM}$ - the inverse relationship between cancer and atherosclerosis dating back to the 1950s which has just recently been confirmed by a cohort study including over 1000 autopsy reports. ${ }^{10,11}$

As the authors acknowledge themselves, there are certain weaknesses of this study that need to be mentioned. Most importantly, limited data on baseline characteristics - specifically regarding the cardiovascular risk profile of the FULIMA cohort-was available, and, as a consequence, no advanced statistical analysis by regression analysis or propensity-score matching could be provided. Therefore, the residual risk for confounding must not be underestimated-particularly since age- and gender-matched controls had no atherosclerotic risk factors or atherosclerotic disease. Patients with MM, in contrast, are known to have a high prevalence of cardiac co-morbidities (as outlined by the authors in their discussion). Furthermore, around half of the patients with MM have renal impairment at presentation, affecting substantially the clearance of $\mathrm{NaF}$ through the kidneys. ${ }^{12,13}$ Consequently, in patients with $\mathrm{MM}$, vascular $\mathrm{NaF}$ uptake might have been increased by a slower renal excretion. Last but not least, the study compares two different cohorts that were scanned with different protocols and on different PET scanners. For all these reasons, it is possible that the higher $\mathrm{NaF}$ uptake in patients with MM could be fully explained by differences in baseline characteristics and acquisition protocols. Nevertheless, the present study emphasizes the potential of NaF PET as a promising technique to amplify our pathophysiological understanding of atherosclerosis and improve the stratification of risk for cardiovascular diseases.

\section{References}

1. Muchtar E, Gertz MA, Kyle RA, Lacy MQ, Dingli D, Leung N, et al. A Modern Primer on light chain amyloidosis in 592 patients with mass spectrometry-verified typing. Mayo Clin Proc 2019;94:472-83.

2. Kistler KD, Kalman J, Sahni G, Murphy B, Werther W, Rajangam $\mathrm{K}$, et al. incidence and risk of cardiac events in patients with previously treated multiple myeloma versus matched patients without multiple myeloma: An observational, retrospective, cohort study. Clin Lymphoma Myeloma Leuk 2017;17:e3.

3. Li W, Garcia D, Cornell RF, Gailani D, Laubach J, Maglio ME, et al. Cardiovascular and thrombotic complications of novel multiple myeloma therapies: A review. JAMA Oncol 2017;3:9808. 
4. Mouhieddine TH, Sperling AS, Redd R, Park J, Leventhal M, Gibson CJ, et al. Clonal hematopoiesis is associated with adverse outcomes in multiple myeloma patients undergoing transplant. Nat Commun 2020;11:2996.

5. Jaiswal S, Natarajan P, Silver AJ, Gibson CJ, Bick AG, Shvartz E, et al. Clonal hematopoiesis and risk of atherosclerotic cardiovascular disease. N Engl J Med 2017;377:111-21.

6. Kwiecinski J, Tzolos E, Adamson PD, Cadet S, Moss AJ, Joshi N, et al. Coronary $18 \mathrm{~F}$-sodium fluoride uptake predicts outcomes in patients with coronary artery disease. J Am Coll Cardiol 2020;75:3061-74.

7. Forsythe RO, Dweck MR, McBride OMB, Vesey AT, Semple SI, Shah ASV, et al. F-sodium fluoride uptake in abdominal aortic aneurysms: The SoFIA. J Am Coll Cardiol 2018;71:513-23.

8. Zirakchian Zadeh M, Østergaard B, Raynor WY, Revheim ME, Seraj SM, Acosta-Montenegro O et al. Comparison of $18 \mathrm{~F}$-sodium fluoride uptake in the whole bone, pelvis, and femoral neck of multiple myeloma patients before and after high-dose therapy and conventional-dose chemotherapy. Eur J Nucl Med Mol Imaging 2020.
9. Blomberg BA, Thomassen A, Takx RA, Vilstrup MH, Hess S, Nielsen AL, et al. Delayed sodium 18F-fluoride PET/CT imaging does not improve quantification of vascular calcification metabolism: Results from the CAMONA study. J Nucl Cardiol 2014;21:293-304

10. Li M, Cima MJ, Milner DA. If it's not one thing, it's another: An inverse relationship of malignancy and atherosclerotic disease. PLoS ONE 2015;10:e0126855.

11. Elkeles A. Cancer and atherosclerosis. Br J Cancer 1956;10:24750 .

12. Yadav P, Cook M, Cockwell P. Current trends of renal impairment in multiple myeloma. Kidney Dis 2016;1:241-57.

13. Czernin J, Satyamurthy N, Schiepers C. Molecular mechanisms of bone 18F-NaF deposition. J Nucl Med 2010;51:1826-9.

Publisher's Note Springer Nature remains neutral with regard to jurisdictional claims in published maps and institutional affiliations. 